\title{
Klebsiella pneumoniae bacteremia and renosplenic abscesses without intestinal symptoms as the initial manifestations of non-steroidal anti-inflammatory drug-induced colitis: a rare case report
}

\author{
Hung-Ling Huang ${ }^{3}$, Po-Liang Lu ${ }^{1,4}$, Chun-Yu Lin ${ }^{1,4}$, Yen-Hsu Chen ${ }^{1,3,4,5}$, Chao-Hung Kuo ${ }^{2}$ and Wei-Ru Lin ${ }^{1 *}$
}

\begin{abstract}
Background: Non-steroidal anti-inflammatory drugs (NSAIDs), the most widely prescribed drugs in the world, can cause gastrointestinal damage, including colitis. However, the prevalence of NSAID-induced colitis is unknown because the disease is often asymptomatic.

Case presentation: We report the case of a 64-year-old female patient with a history of long-term NSAID use, who was hospitalized with septic shock caused by Klebsiella pneumoniae bacteremia. Computed tomography revealed multiple renal and splenic abscesses with diffuse colon wall thickening. A colonoscopy confirmed colitis with diffuse ulcers. NSAIDs were discontinued after this hospitalization. The abscesses improved after antibiotic treatment. A short course of balsalazide treatment was given under the suspicion of ulcerative colitis. Balsalazide was discontinued four months later due to a non-compatible clinical course. A follow-up colonoscopy two years later revealed a normal colon mucosa, and NSAID-induced colitis was diagnosed.

Conclusion: This is the first reported case of combined bacterial splenic and renal abscesses without intestinal manifestations as the initial presentation of NSAID-induced colitis. In contrast to cases of K. pneumoniae bacteremia with primary liver abscesses in patients with diabetes mellitus in Taiwan, we presented the first case with abscesses caused by community-acquired $K$. pneumoniae in the kidneys and spleen without liver invasion. In conclusion, our case report alerts clinicians to the possibility that $K$. pneumoniae bacteremia combined with multiple abscesses can be associated with severe NSAID-induced colitis.
\end{abstract}

Keywords: Non-steroidal anti-inflammatory drugs, Colitis, Bacteremia, Klebsiella pneumoniae, Renosplenic abscesses

\section{Background}

Non-steroidal anti-inflammatory drugs (NSAIDs), despite their well-known adverse effects on the gastrointestinal (GI) tract, are widely prescribed worldwide. NSAIDs damage different regions of the GI tract, including the distal small bowel and colon can be the target of NSAIDs. A study using fecal calprotectin (a non-degraded neutrophil cytosolic protein) to diagnose NSAID enteropathy

\footnotetext{
*Correspondence: 940268@ms.kmuh.org.tw

'Division of Infectious Diseases, Department of Internal Medicine, Kaohsiung Medical University Hospital, Kaohsiung Medical University, 100, Tzyou 1st Road, Kaohsiung 807, Taiwan

Full list of author information is available at the end of the article
}

found that the prevalence of NSAID enteropathy was around 44\% [1]. Studies evaluating the colonic side effects of NSAIDs have suggested that NSAID-induced colitis is common; however, symptomatic NSAIDinduced colitis is rare [2,3]. People taking NSAIDs are two to five times more likely to develop colonic inflammation than the general population. NSAIDs affect the large intestine by causing colonic ulceration and stricture formation. Approximately $10 \%$ of newly diagnosed colitis cases may be related to NSAID administration $[4,5]$. However, the prevalence of NSAID-induced colitis is unknown because the disease is often asymptomatic. 
NSAID-induced colitis usually has nonspecific histological findings. The diagnosis of NSAID-induced colonic ulceration has been made based on a history of NSAID use and the exclusion of other causes [2]. The temporal relationship between NSAID use and symptoms that resolve after cessation confirm the diagnosis of NSAID-induced colitis.

Intra-abdominal abscesses as the initial presentation of NSAID-induced colitis has not been reported previously. Herein, we report the unusual case of a patient with renal and splenic abscesses but without intestinal symptom as the initial manifestations of NSAIDinduced colitis.

\section{Case presentation}

A 64-year-old Taiwanese woman presented with a history of well-controlled type 2 diabetes mellitus and hypertension. She had been self-treating regularly with NSAIDs from pharmacy (diclofenac, $50 \mathrm{mg}$ three times daily) for more than two years because of chronic low back pain caused by intervertebral disc herniation. General malaise, poor appetite, intermittent postprandial nausea, urinary urgency and frequency were present one month before admission. She visited local clinics and received no specific diagnosis. Due to progressive shortness of breath and drowsiness for one week, she was sent to a medical center. She appeared acutely ill but afebrile, with a blood pressure of $74 / 34 \mathrm{mmHg}$, a pulse rate of $86 / \mathrm{min}$, a respiratory rate of $22 / \mathrm{min}$, and an oxygen saturation level of $93 \%$ in ambient air. Physical examination revealed no specific local findings. A blood assay revealed the following findings: leukocyte count, $44,400 / \mu \mathrm{L}$ with $85 \%$ neutrophils; hemoglobin, $12.3 \mathrm{~g} / \mathrm{dL}$; platelet count, 466, $000 / \mu \mathrm{L}$; C-reactive protein, $235.76 \mathrm{mg} / \mathrm{L}$; total bilirubin, $1.17 \mathrm{mg} / \mathrm{dL}$; direct bilirubin, $0.17 \mathrm{mg} / \mathrm{dL}$; aspartate aminotransferase, $38 \mathrm{IU} / \mathrm{L}$; alanine aminotransferase, $21 \mathrm{IU} / \mathrm{L}$; blood urea nitrogen, $96.77 \mathrm{mg} / \mathrm{dL}$; creatinine, $7.8 \mathrm{mg} / \mathrm{dL}$; sodium, $140 \mathrm{mmol} / \mathrm{L}$; potassium, $8.6 \mathrm{mmol} / \mathrm{L}$; and lactate, $20.3 \mathrm{mmol} / \mathrm{L}$. The arterial blood gas analysis revealed severe metabolic acidosis ( $\left.\mathrm{pH}, 7.02 ; \mathrm{HCO}_{3}, 7.1 \mathrm{mmol} / \mathrm{L}\right)$. A chest radiograph revealed no pulmonary lesions. Urine analysis via catheterization revealed only mild hematuria.

She was admitted to the intensive care unit due to septic shock, acute respiratory failure, acute renal failure, and hyperkalemia. She received mechanical ventilation, intravenous vasopressors, continuous venovenous hemofiltration, and cefmetazole ( $2 \mathrm{~g} /$ day $)$ was prescribed empirically. All blood cultures yielded K. pneumoniae. The urine culture was sterile. Contrastenhanced abdominal computed tomography (CT) revealed multiple abscesses in the spleen and bilateral kidneys, with the largest lesion (approximately $5.2 \mathrm{~cm}$ in diameter) located in the right kidney (Figure 1). In addition, CT also revealed segmental bowel wall edema in the submucosal layer over the terminal ileum, cecum, ascending colon, and descending colon with perifocal fat stranding. The hyperemic change was compatible with the inflammatory reaction in the bowel. The CT also revealed more intense wall enhancement at the terminal ileum (Figure 2). A colonoscopy revealed diffuse ulceration with exudation in the distal terminal ileum and segmental ulceration in the colon, with a reduction in the lumen caliber in the sigmoid colon (Figure 3). The pathological analysis of colon biopsy specimens revealed ulcers, necrotic debris, and the infiltration of lymphocytes into the edematous lamina propria (Figure 4). Three weeks later, a follow-up abdominal ultrasonography demonstrated partial resolution of the renal and splenic abscesses.

The patient was treated in the outpatient department with $500 \mathrm{mg}$ of oral cephradine, four times daily; $500 \mathrm{mg}$ of metronidazole, three times daily; and balsalazide (2.25 g daily) for two months. The follow-up abdominal CT scan revealed complete resolution of the abscesses two months later, and the colonoscopy revealed multiple segmental longitudinal ulceration with skip lesions in the rectum, sigmoid, and terminal ileum. A colon biopsy showed necrotizing inflammation with the infiltration of mixed inflammatory cells ,including neutrophils, histiocytes, and lymphocytes. Moreover, focal ulcerated colonic mucosa with inflammatory infiltration was found in the edematous, fibrous lamina propria. Some distorted glands were observed, but no crypt abscesses were noted. Balsalazide was discontinued after a course of four months. She had an uneventful course in the out-patient department, and a follow-up colonoscopy two years later revealed melanosis coli and internal hemorrhoid only, without ulcers or stenosis over the entire colon.

\section{Discussion}

Our case is unique due to the presentation of severe colitis without an initial manifestation of intestinal symptoms. NSAID-induced colitis was diagnosed based on the history of long-term NSAID use after excluding other etiologies. To the best of our knowledge, this is the first reported case of NSAID-induced colitis with renal and splenic abscesses caused by communityacquired $K$. pneumoniae without liver involvement.

K. pneumoniae, a gram-negative encapsulated aerobic bacterium, is part of the normal flora of the human mouth and intestines. Human carrier rates in the community range from 5 to $38 \%$ in the stool and from 1 to $6 \%$ in the nasopharynx [6,7]. The infection rate is higher for individuals with impaired host defenses, such as individuals with diabetes mellitus and hepatobiliary tract disease $[8,9]$. K. pneumoniae has been reported to be the most common pathogen associated with pyogenic hepatic abscesses and splenic abscesses, especially in Taiwan 


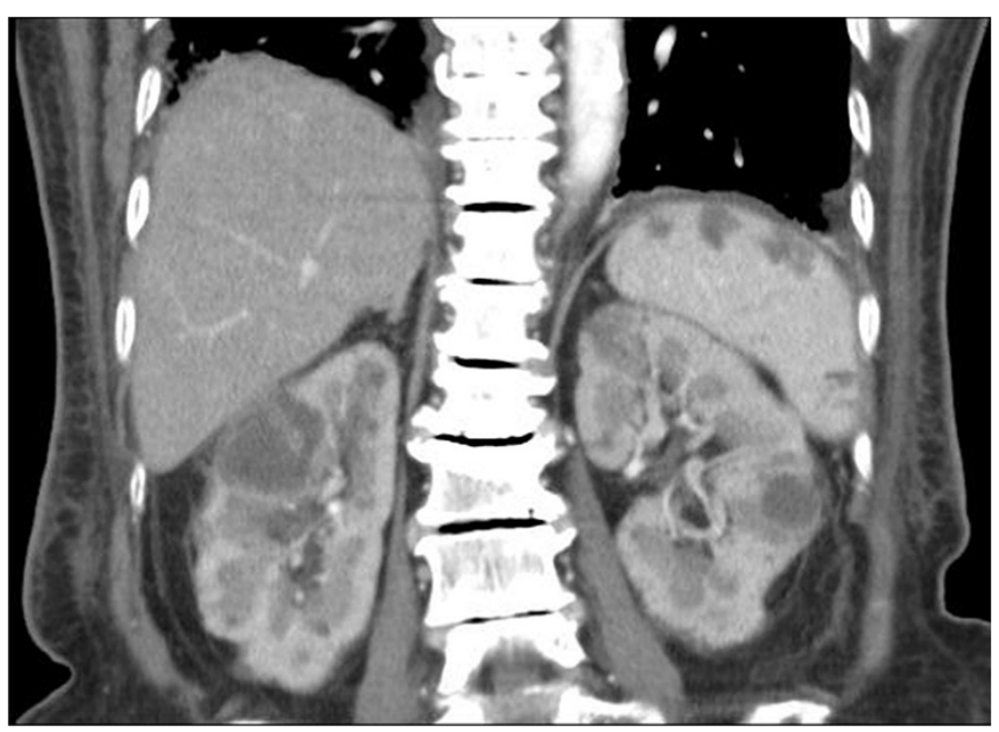

Figure 1 Computed tomography scans of the abdomen revealing several rim-enhancing hypodense lesions in bilateral kidneys and the spleen.

and countries in Eastern and Southeast Asia [10-12], and this bacterium accounts for $10-16 \%$ of cases of splenic abscess and approximately $25 \%$ of cases of renal abscess $[13,14]$.

Intra-abdominal abscesses have diverse symptoms. Fever and abdominal pain are the most frequent symptoms, but a few cases have vague symptoms, as in the case presented herein. Imaging analysis, including ultrasonography and computed tomography $(\mathrm{CT})$, are necessary to diagnose intra-abdominal infections [15].

Splenic abscesses are rare, with a reported frequency of $0.14-0.7 \%$ in case reports [16]. Chang et al. analyzed 67 splenic abscess cases which occurred over a period of
19 years and found that $K$. pneumoniae was the most frequently encountered pathogen in blood cultures or abscess cultures, similar to observations from other Asian countries [11]. Ferraioli G. et al. found a different result; they observed that the most common pathogens were polymicrobial pathogens in pus cultures and Staphylococcus species in blood cultures [16].

The pathogenesis of intra-abdominal abscesses caused by $K$. pneumoniae remains unclear. One hypothesis for the pathogenesis of pyogenic intra-abdominal abscesses involves hematogenous bacterial seeding from the GI tract. An animal study demonstrated that K. pneumoniae strains with genetic regulatory networks for translocation

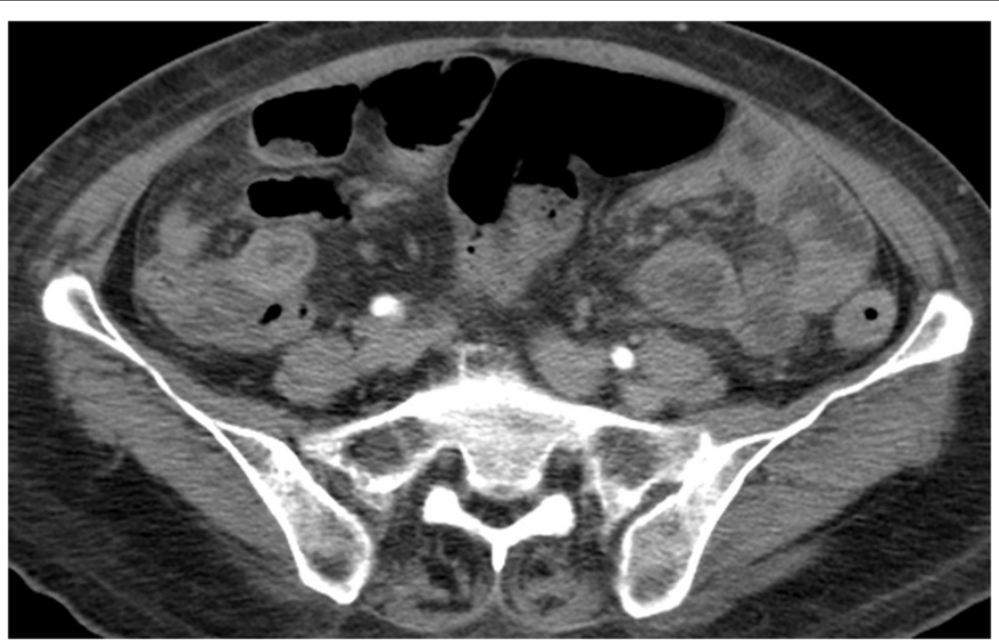

Figure 2 Computed axial tomography scans of the abdomen showing marked thickening of the wall of the terminal ileum, cecum, ascending colon, descending colon, and rectum with perifocal fat stranding. These finding are compatible with the findings of the colonoscopy. 


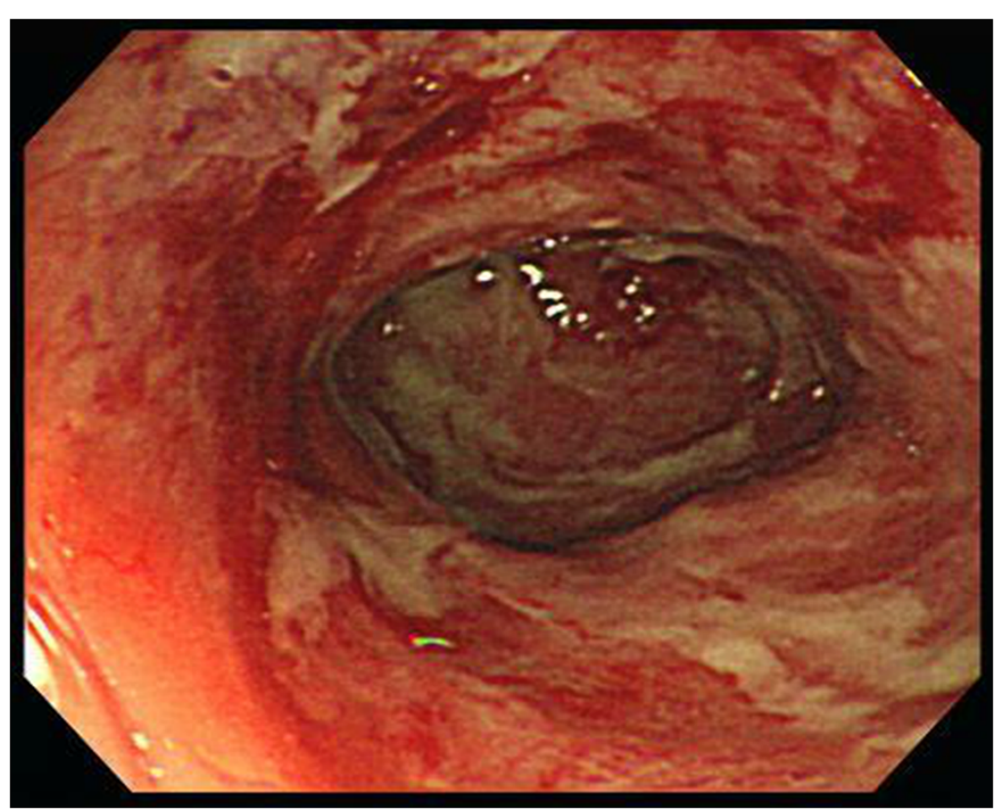

Figure 3 A colonoscopy revealed extensive segmental longitudinal ulceration of the mucosa extending over the rectum, sigmoid colon, and terminal ileum.

have the ability to cross the intestinal barrier [17]. A careful search for the source of $K$. pneumoniae bacteremia other than primary bacteremia should be considered, and which should include colon lesions survey [18]. In our case, the finding of thickened colonic wall prompted us to consider the colon as the possible source of $K$. pneumoniae bacteremia.

Prostaglandins represent one of the most important components of mucosal defense in the colon and are involved in the maintenance of microcirculation and blood flow to modulate the mucosal immune system. NSAIDs inhibit colonic prostaglandin synthesis, leading to the development of colitis and the aggravation of preexisting intestinal diseases.

The ileocecal region is the most common site of NSAID-induced colonic injury. Pathological examination of colon specimens from patients with NSAID-induced colitis usually reveals sharply demarcated, semilunar or

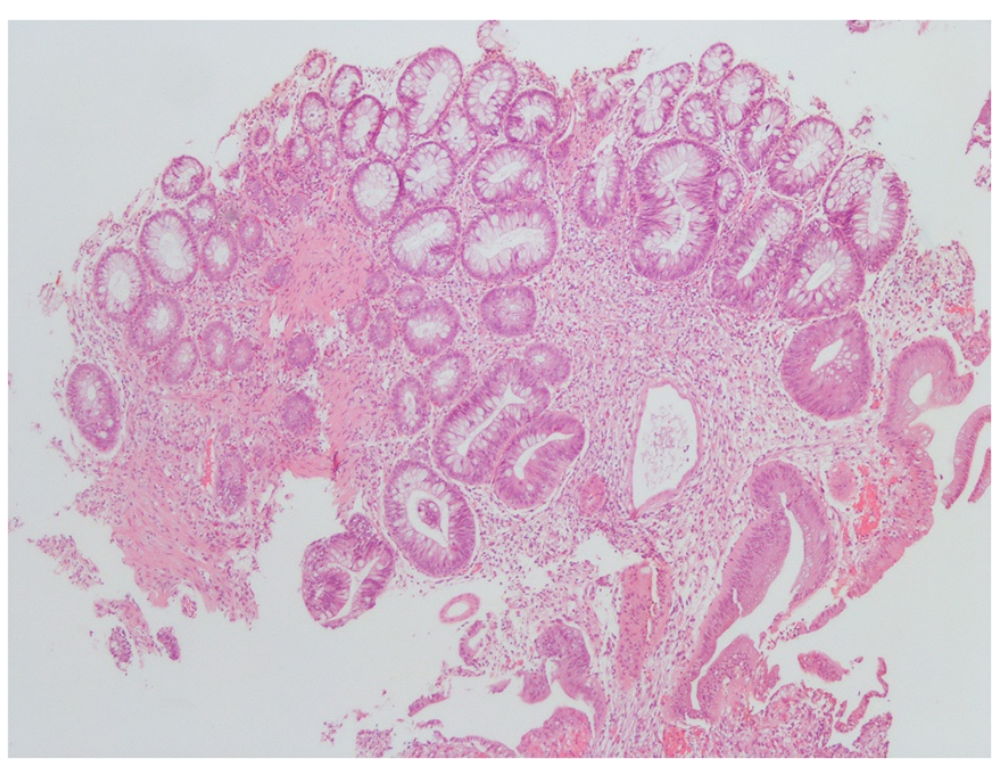

Figure 4 Photomicrograph of a sigmoid colon biopsy specimen displaying an ulcer, necrotic debris, and lymphocyte infiltration into the edematous lamina propria (hematoxylin and eosin, original magnification $\times 100$ ). 
circumferential, superficial ulcerations with normal surrounding mucosa. There are no specific histopathological findings for NSAID-induced colitis except for the diaphragm-like stricture. Cellular infiltration into the lamina propria and epithelium may involve predominantly neutrophils or lymphocytes, depending on the chronicity of the lesion [19]. Symptomatic patients may have watery or bloody diarrhea, weight loss, fatigue, abdominal pain, anorexia, iron-deficiency anemia, and hypoalbuminemia.

There have been no studies to determine the risk of colitis related to different types of NSAIDs; however, many reports have stated that diclofenac, mefenemic acid, piroxicam, and ibuprofen could cause colorectal ulcers or colitis [4,20-22]. According to one case-controlled study, the long-term use of NSAIDs increased the risk of colonic mucosal lesions, suggesting that NSAIDs may contribute to the pathogenesis of colonic ulcers or colitis [23]. In addition, naproxen has been implicated in eosinophilic colitis [24], and diclofenac may be associated with pseudomembranous colitis [25].

In this case, the colonoscopy findings and pathological findings were not able to determine the etiology of the colitis. Due to evidence of a possible association between the use of NSAIDs and the exacerbation or relapse of inflammatory bowel diseases [26,27], NSAID-exacerbated inflammatory bowel disease was initially suspected. This patient was initially treated with balsalazide, but she became stable despite the discontinuation of balsalazide.

The discontinuation of NSAIDs is the cornerstone of the treatment of NSAID-induced colitis. Symptoms usually resolve within days to weeks of the withdrawal of NSAIDs, with the restoration of normal histology. Our patient received a colonoscopy two years later that revealed complete restoration of the colon mucosa; thus, this patient was diagnosed with NSAID-induced colitis.

\section{Conclusions}

We present a unique case of NSAID-induced colitis without any GI symptoms at initial presentation, that was associated with septic shock caused by $K$. pneumoniae bacteremia with multiple renal and splenic abscesses. The colitis was found incidentally by CT and then identified as NSAID-induced colitis. This is also the first reported case of disseminated abscess formation caused by community-acquired $K$. pneumoniae in the kidneys and spleen without liver involvement in an NSAIDinduced colitis patient.

This case is a reminder that hematogenous intraabdominal abscesses caused by K. pneumoniae necessitate a thorough investigation of the GI tract. Assessing the presence of colitis should be considered even in the absence of preceding gastrointestinal symptoms, especially for those who have previously used NSAIDs.

\section{Consent}

Written informed consent was obtained from the patient for publication of this case report and any accompanying images. A copy of the written consent is available for review by the Editor of this journal.

\section{Abbreviations \\ NSAIDs: Non-steroidal anti-inflammatory drugs; Gl: Gastrointestinal; \\ CT: Computed tomography.}

\section{Competing interests}

The authors declared that they have no competing interests.

\section{Authors' contributions}

HLH, WRL, and PLL prepared the manuscript. YHC and CHK provided laboratory support. WRL and CHK cared for the patient and provided advice on the clinical aspects of the case report. All authors read and approved the final version of the manuscript.

\section{Author details}

'Division of Infectious Diseases, Department of Internal Medicine, Kaohsiung Medical University Hospital, Kaohsiung Medical University, 100, Tzyou 1st Road, Kaohsiung 807, Taiwan. ${ }^{2}$ Division of Gastroenterology, Department of Internal Medicine, Kaohsiung Medical University Hospital, Kaohsiung Medical University, Kaohsiung, Taiwan. ${ }^{3}$ Department of Internal Medicine, Kaohsiung Medical University Hospital, Kaohsiung Medical University, Kaohsiung, Taiwan. ${ }^{4}$ School of Medicine, College of Medicine, Kaohsiung Medical University, Kaohsiung, Taiwan. ${ }^{5}$ Graduate Institute of Medicine, College of Medicine, Kaohsiung Medical University, Kaohsiung, Taiwan.

Received: 26 April 2013 Accepted: 20 September 2013

Published: 22 September 2013

\section{References}

1. Deshpande V, Hsu M, Kumarasinghe MP, Lauwers GY: The clinical significance of incidental chronic colitis: a study of 17 cases. Am J Surg Pathol 2010, 34:463-469.

2. Kurahara K, Matsumoto T, lida M, Honda K, Yao T, Fujishima M: Clinical and endoscopic features of nonsteroidal anti-inflammatory drug-induced colonic ulcerations. Am J Gastroenterol 2001, 96:473-480.

3. Puspok A, Kiener HP, Oberhuber G: Clinical, endoscopic, and histologic spectrum of nonsteroidal anti-inflammatory drug-induced lesions in the colon. Dis Colon Rectum 2000, 43:685-691.

4. Tanner AR, Raghunath AS: Colonic inflammation and nonsteroidal antiinflammatory drug administration. An assessment of the frequency of the problem. Digestion 1988, 41:116-120.

5. Katsinelos P, Christodoulou K, Pilpilidis I, Xiarchos P, Papagiannis A Dimiropoulos S, Amperiadis P, Vasiliadis T, Tarpagos A, Katsos I, Eugenidis N: Colopathy associated with the systemic use of nonsteroidal antiinflammatory medications. An underestimated entity. Hepatogastroenterology 2002, 49:345-348.

6. Fung CP, Lin YT, Lin JC, Chen TL, Yeh KM, Chang FY, Chuang HC, Wu HS, Tseng CP, Siu LK: Klebsiella pneumoniae in gastrointestinal tract and pyogenic liver abscess. Emerg Infect Dis 2012, 18:1322-1325.

7. Podschun R, Ullmann U: Klebsiella spp. as nosocomial pathogens: epidemiology, taxonomy, typing methods, and pathogenicity factors. Clin Microbiol Rev 1998, 11:589-603.

8. Kang Cl, Kim SH, Bang JW, Kim HB, Kim NJ, Kim EC, Oh MD, Choe KW: Community-acquired versus nosocomial Klebsiella pneumoniae bacteremia: clinical features, treatment outcomes, and clinical implication of antimicrobial resistance. J Korean Med Sci 2006, 21:816-822.

9. Wu HS, Wang FD, Tseng CP, Wu TH, Lin YT, Fung CP: Characteristics of healthcare-associated and community-acquired Klebsiella pneumoniae bacteremia in Taiwan. $J$ Infect 2012, 64:162-168,

10. Lederman ER, Crum NF: Pyogenic liver abscess with a focus on Klebsiella pneumoniae as a primary pathogen: an emerging disease with unique clinical characteristics. Am J Gastroenterol 2005, 100:322-331.

11. Chang KC, Chuah SK, Changchien CS, Tsai TL, Lu SN, Chiu YC, Chen YS, Wang CC, Lin JW, Lee CM, Hu TH: Clinical characteristics and prognostic 
factors of splenic abscess: a review of 67 cases in a single medical center of Taiwan. World J Gastroenterol 2006, 12:460-464.

12. Siu LK, Yeh KM, Lin JC, Fung CP, Chang FY: Klebsiella pneumoniae liver abscess: a new invasive syndrome. Lancet Infect Dis 2012, 12:881-887.

13. Lee CH, Leu HS, Hu TH, Liu JW: Splenic abscess in southern Taiwan. J Microbiol Immunol Infect 2004, 37:39-44.

14. Lee BE, Seol HY, Kim TK, Seong EY, Song SH, Lee DW, Lee SB, Kwak IS: Recent clinical overview of renal and perirenal abscesses in 56 consecutive cases. Korean J Intern Med 2008, 23:140-148.

15. Solomkin JS, Mazuski JE, Bradley JS, Rodvold KA, Goldstein EJ, Baron EJ, O'Neill PJ, Chow AW, Dellinger EP, Eachempati SR, et al: Diagnosis and management of complicated intra-abdominal infection in adults and children: guidelines by the Surgical Infection Society and the Infectious Diseases Society of America. Clin Infect Dis 2010, 50:133-164.

16. Ferraioli G, Brunetti E, Gulizia R, Mariani G, Marone P, Filice C: Management of splenic abscess: report on 16 cases from a single center. Int J Infect Dis 2009, 13:524-530.

17. Tu YC, Lu MC, Chiang MK, Huang SP, Peng HL, Chang HY, Jan MS, Lai YC: Genetic requirements for Klebsiella pneumoniae-induced liver abscess in an oral infection model. Infect Immun 2009, 77:2657-2671.

18. Jeong SW, Jang JY, Lee TH, Kim HG, Hong SW, Park SH, Kim SG, Cheon YK, Kim YS, Cho YD, et al: Cryptogenic pyogenic liver abscess as the herald of colon cancer. J Gastroenterol Hepatol 2012, 27:248-255.

19. Goldstein NS, Cinenza AN: The histopathology of nonsteroidal antiinflammatory drug-associated colitis. Am J Clin Pathol 1998, 110:622-628.

20. Ravi S, Keat A, Keat E: Colitis caused by non-steroidal anti-inflammatory drugs. Postgrad Med J 1986, 62:773-776.

21. Witham R: Voltaren (diclofenac sodium)-induced ileocolitis. Am J Gastroenterol 1991, 86:246-247.

22. Pan YS, Chen LT, Tseng CA, Su YC, Jan CM, Wang WM, Tsai KB: Clinical and endoscopic features of non-steroidal anti-inflammatory drug-induced colorectal ulcerations. J Formos Med Assoc 2005, 104:804-810.

23. Shibuya T, Ohkusa T, Yokoyama T, Harada A, Beppu K, Sakamoto N, Osada T, Nagahara A, Terai T, Otaka M: Colonic mucosal lesions associated with long-term or short-term administration of nonsteroidal antiinflammatory drugs. Colorectal Dis 2010, 12:1113-1121.

24. Alfadda AA, Storr MA, Shaffer EA: Eosinophilic colitis: epidemiology, clinical features, and current management. Therap Adv Gastroenterol 2011, 4:301-309.

25. Gentric A, Pennec Y: Diclofenac-induced pseudomembranous colitis. Lancet 1992, 340:126-127.

26. Ballinger A: Adverse effects of nonsteroidal anti-inflammatory drugs on the colon. Curr Gastroenterol Rep 2008, 10:485-489.

27. Kefalakes H, Stylianides TJ, Amanakis G, Kolios G: Exacerbation of inflammatory bowel diseases associated with the use of nonsteroidal anti-inflammatory drugs: myth or reality? Eur J Clin Pharmacol 2009, 65:963-970

doi:10.1186/1471-230X-13-139

Cite this article as: Huang et al.: Klebsiella pneumoniae bacteremia and renosplenic abscesses without intestinal symptoms as the initial manifestations of non-steroidal

anti-inflammatory drug-induced colitis: a rare case report. $B M C$ Gastroenterology 2013 13:139.

\section{Submit your next manuscript to BioMed Central and take full advantage of:}

- Convenient online submission

- Thorough peer review

- No space constraints or color figure charges

- Immediate publication on acceptance

- Inclusion in PubMed, CAS, Scopus and Google Scholar

- Research which is freely available for redistribution

Submit your manuscript at www.biomedcentral.com/submit
C Biomed Central 